\title{
THE SMALLEST ELECTRET MICROPHONE WITH GOOD PERFORMANCE
}

\author{
Aart van Halteren ${ }^{1}$, Dion de Roo ${ }^{1}$, Lucas Evers ${ }^{2}$
}

${ }^{1}$ Sonion Nederland B.V., Taurusavenue 143, 2132LS Hoofddorp

Sonion Vietnam, Lot I3-9, Saigon Hi-Tech Park, District 9, Ho Chi Minh City, Vietnam

\begin{abstract}
A high performance miniature electret microphone
In the field of Hearing Aids the most important parameter for a microphone is performance versus size. This is not a simple parameter like Signal to Noise ratio - versus volume, but the size is strongly connected to the overall space used in the Hearing Instrument. Especially because in most hearing aids two microphones are used, in a matched pair configuration, to create directionality.
\end{abstract}

Power consumption is always a limiting factor in these kinds of applications. We will present tradeoffs and results for the different parameters, and design options to come to the final design that can be produced in an industrial way.

Key words: Electret, Microphone, Hearing, Noise, Directionality

\section{A high performance miniature electret microphone}

Until now the biggest volume of microphones sold to be used in Hearing Aids were made in a squared shape. Going to a rectangular shape presents new challenges on how to acoustically model and optimize such a microphone. But the rectangular shape also allows you to make interesting combinations, which makes it possible to optimize the mechanical design of the hearing aid with two matched microphones, or even better to make a integrated module of matched microphones. Microphones for hearing aids were originally designed to be Omnidirectional and were optimized for that. At a certain point in history, the audio-logical advantages from using directionality became very apparent. And a matched pair was made based on two microphones. There were two apparent disadvantages: the microphones were not designed to be a matched pair, so the only way to get them matched was a selection process in the production, the other disadvantage being that both microphones drifted over time, making it very difficult to keep the directionality stable over time. Many smart algorithms were defined to solve this, but it would of course be very advantageous if the microphones itself could do this. An obvious choice would be to use MEMS microphones, which are very stable, and which have improved significantly over time in noise performance, but electret microphones are still better. In hearing aids the noise performance is of prime importance. By a clever construction you can actually optimize the performance in a module specially designed for directional performance in the smallest possible size.

\section{A more detailed analysis of the problem}

To make directional microphones using two omni microphones you use a block diagram as shown below : Dual Microphone Directional System

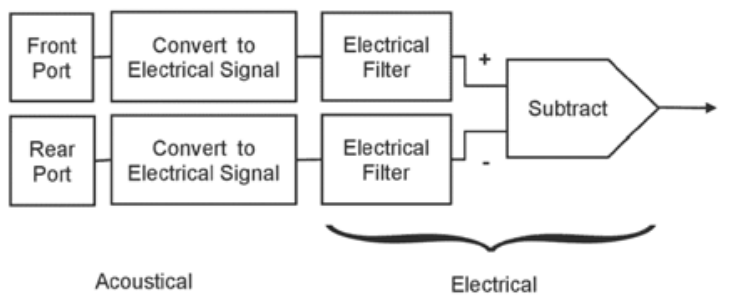

Fig. 1 : AMA Sensors 2013_how to make a directional microphone out of two omni-directional microphones.

The electrical part can incorporate a delay, which determines the directional characteristic of the combination. Without a delay you get a polar response that looks like a figure eight : if both microphones get the same signal (so coming from the side) there is no output. Since you substract the signals you get less signal and the noise is uncorrelated so you get $3 \mathrm{~dB}$ noise increase. If the port spacing is bigger, the signals differ more and you get 
more signal, improving the signal to noise ratio.

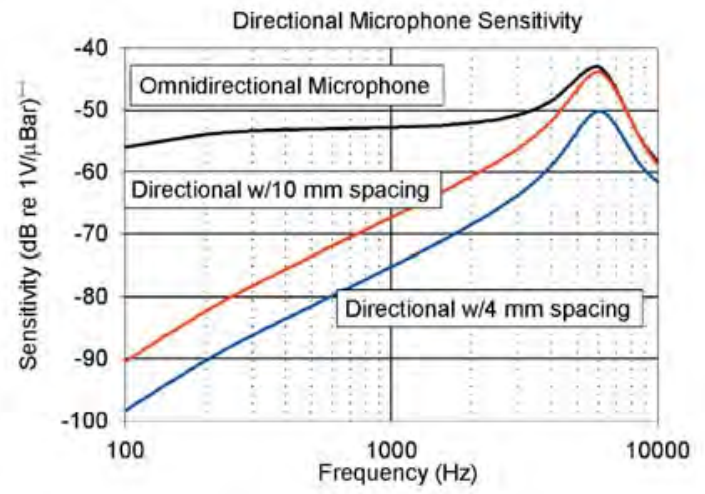

Fig. 2 : AMA Sensors 2013_Different frequency characteristics of microphones.

Today the most popular hearing aid is the Behind The Ear style (BTE). Where roughly two variants exist: one with the receiver behind the ear and one type where the receiver is in the ear. (Receiver In the Canal, RIC style). One of the main design problems for hearing aids is that people don't want to show that they have a hearing aid, which means that smaller in size is almost always better. For directional microphones the Signal to Noise ratio linearly increases by increasing the distance between the two omni-directional microphones. So this is an interesting tradeoff: on one hand you want a small port spacing to make the hearing aid smaller, and on the other hand you want to make the port spacing as big as possible to get a better Signal to Noise ratio. There is one other problem associated with bigger port spacing on a Behind the Ear Hearing Aid: there is fewer signal if you move the microphone more backwards (due to the shadow effect of you head) and you still want to look at a horizontal angle and not upwards, so there are limits because of that too.

\section{Microphone Size versus Signal to Noise} ratio

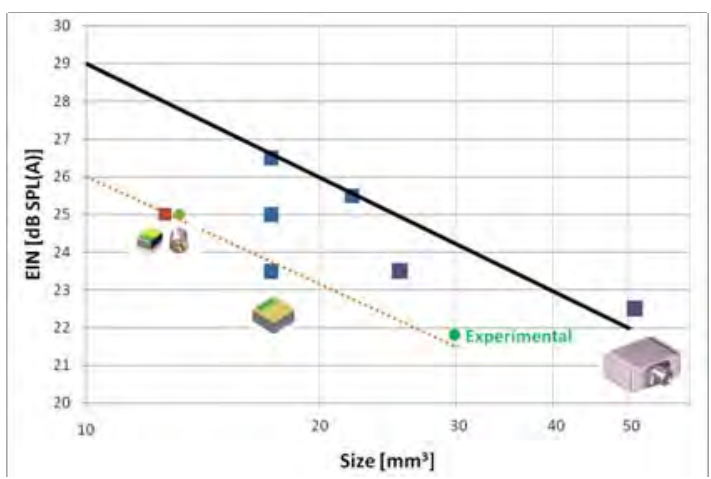

Fig. 3 : AMA Sensors 2013_Equivalent input noise vs. size in miniature electrets microphones for different design tradeoffs.

So to get a good signal to noise ratio, you need a big microphone, with big port spacing, but you want the smallest package for cosmetic reasons in the Hearing Aid. But that is still not the whole story, because you can still have different shape factors to optimize the use of the space in the hearing aid. In general if with a rectangular shape there is more design freedom, compared to the traditional squarely shaped microphones.
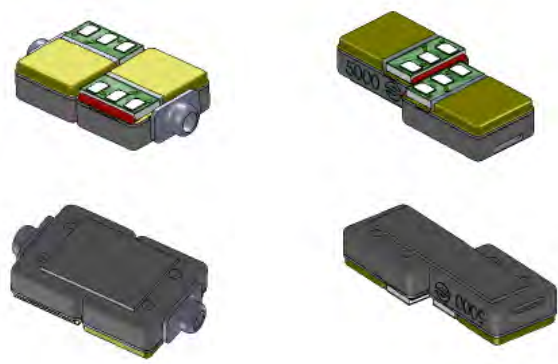

Fig. 4 : AMA Sensors 2013_combining Rectangular microphones

Although changing to rectangular worked well, we became wiser in the mean time and combined everything in one microphone module

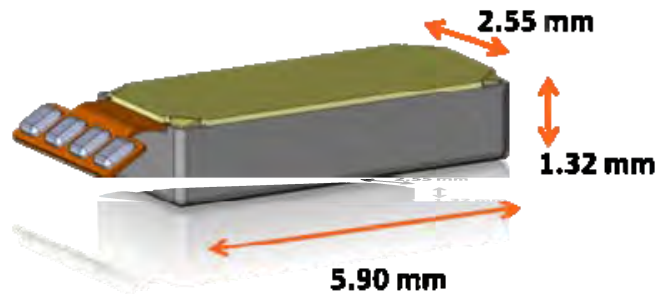

Fig. 5 : AMA Sensors 2013_Making one microphone module

This fits very nicely in a typical BTE

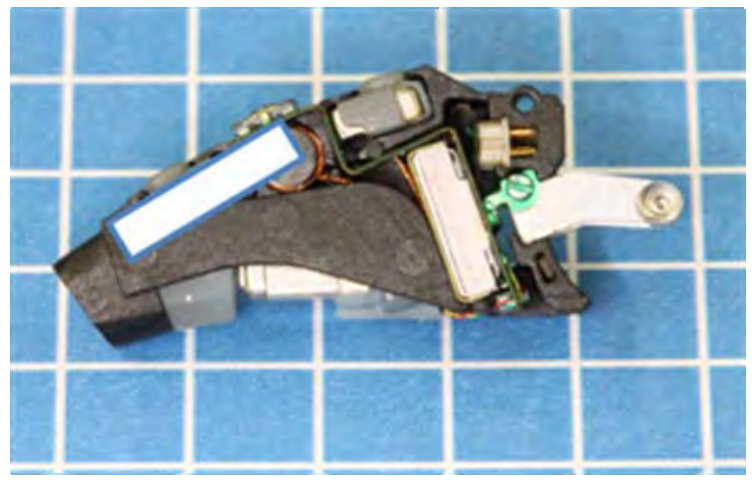

Fig. 6 : AMA Sensors 2013 Fitting of the module in a BTE Hearing Instrument.

But what really makes the difference is that combining these microphones in one module makes a design possible that is optimized for Directional performance and not for Omni performance. Today when hearing aids are in a quiet environment they switch to Omni mode, taking away the possibility to get rid of disturbing signals, that might also be there in a quiet environment. The reason is that the noise in directional mode is too high. 


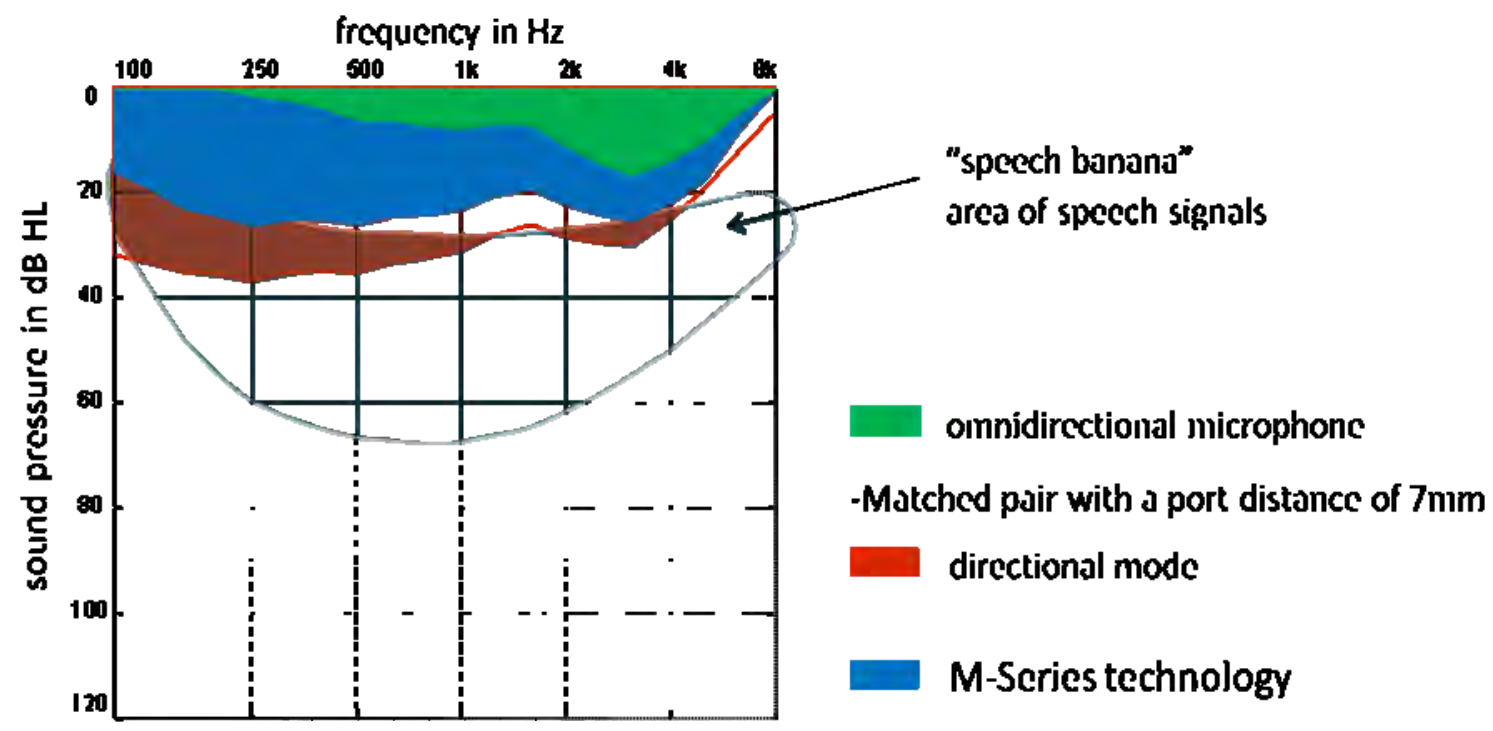

So in the traditional technology you see that the normal area of speech signals is completely free of noise in the

omni mode (green) but overlapping in directional mode (red). The noise of the new module

Fig. 7 : AMA Sensors 2013 Speech spectrum versus noise.

in ,M-technolgy' is sketched in blue, and does not overlap the levels of normal speech at $65 \mathrm{~dB}$ SPL.

\section{Summary}

By optimizing the Shape of the microphones, optimizing for the smallest size and combining for directional performance the best tradeoff between size, directional and noise performance in a hearing aid can be achieved. 